\title{
Simulation via instant messaging - Birmingham advance (SIMBA): an innovative simulation-based learning model that helped to keep medical education continue during the COVID-19 pandemic
}

\author{
Authors: Georgia Morgan, ${ }^{\mathrm{A}}$ Emma Ooi, ${ }^{\mathrm{B}}$ Thia Hanania, ${ }^{\mathrm{C}}$ Meri Davitadze, ${ }^{\mathrm{D}}$ Dengyi Zhou, ${ }^{\mathrm{C}}$ Parisha Blaggan, ${ }^{\mathrm{C}}$ \\ Eka Melson, ${ }^{\mathrm{E}}$ Wiebke Arlt, ${ }^{\mathrm{E}}$ Kristien Boelaert, ${ }^{\mathrm{F}}$ Rachel Cooney, ${ }^{\mathrm{G}}$ Shri Pathmakanthan, ${ }^{\mathrm{G}}$ Vinay Reddy-Kolanu, ${ }^{\mathrm{G}}$ \\ Niki Karavitaki, ${ }^{\mathrm{E}}$ Cai Ying Ng, ${ }^{\mathrm{B}}$ Isabel Allison, ${ }^{\mathrm{C}}$ Wentin Chen, ${ }^{\mathrm{C}}$ Lucretia Thomas, ${ }^{\mathrm{C}}$ Nia Evans ${ }^{\mathrm{H}}$ and Punith Kempegowda ${ }^{\mathrm{E}}$
}

\section{Introduction}

In response to COVID-19, the delivery of medical education has largely transitioned from face-to-face teaching to virtual platforms. Simulation-based learning is a useful teaching modality to develop clinicians' knowledge and skills, while protecting patients from harm. ${ }^{1}$ While simulation has traditionally occurred via face-to-face role play, many of its principles can be adapted for remote learning. The aim of this study was to explore the effectiveness of Simulation via Instant Messaging - Birmingham Advance (SIMBA) as a model of virtual simulation-based medical education during the COVID-19 pandemic.

\section{Materials and methods}

Five SIMBA sessions were conducted between May 2020 and August 2020. Each session focused on a particular topic: adrenal pathology, thyroid pathology, pituitary pathology, inflammatory bowel disease and acute medical pathologies. In each session, five standardised transcripts based on real-life anonymised clinical data were used to simulate clinical cases. During the simulation, participants interacted with moderators (mostly medical students) through WhatsApp. For each case, participants were asked to assess the patient as they would in real life and formulate a diagnosis and management plan. This was followed by an interactive discussion session with an expert in their respective field, delivered via Zoom. Detailed

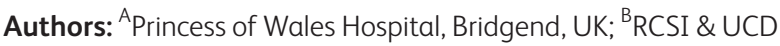
Malaysia Campus, Malaysia; ${ }^{C}$ College of Medical and Dental Sciences, Birmingham, UK; ${ }^{D}$ Georgia-American Family Medicine Clinic (Medical House), Tbilisi, Georgia; ${ }^{E}$ Institute of Metabolism

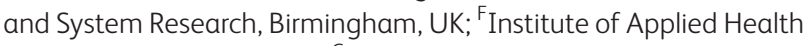
Research, Birmingham, UK; ${ }^{G}$ Queen Elizabeth Hospital, Birmingham, UK; ${ }^{H}$ Royal Glamorgan Hospital, Rhondda Cynon Taf, UK methodology for SIMBA was recently published. ${ }^{2}$ Wilcoxon signed rank test was used to investigate the effect of SIMBA sessions on self-reported confidence levels of participants' approach to clinical scenarios pre- and post-SIMBA on Likert scale. We also analysed the acceptance and relevance of the simulated cases by participants and results are described in frequencies and proportions as appropriate.

\section{Results and discussion}

204 participants completed both pre- and post-SIMBA evaluation forms and were included in the analysis. 129 participants were from the UK and 75 participants were from rest of the world. There was a significant improvement in selfreported confidence in participants' approach to the simulated cases (overall (204; $<<0.001)$; adrenal (33; $<<0.001)$; thyroid (37; $<<0.001)$; pituitary (79; $<<0.001)$; inflammatory bowel disease (17; $<<0.001)$; acute medicine $(38 ; \mathrm{p}<0.001)) .94 .6 \%$ $(193 / 204)$ strongly agreed/agreed the simulated topics were applicable to their clinical practice. $89.7 \%$ (183/204) strongly agreed/agreed SIMBA sessions accommodated their personal learning style and $92.6 \%(189 / 204)$ strongly/agreed the sessions were engaging. 94.6\% (193/204) and 94.1\% (192/204) strongly agreed/agreed the content was impactful at both a personal level and a professional level. Participants felt that SIMBA improved their clinical competencies in the following areas: $52.0 \%(106 / 204)$ patient care, $30.9 \%(63 / 204)$ professionalism, $84.8 \%$ (173/204) knowledge on patient management, $48.0 \%$ (98/204) systems-based practice, $69.6 \%(142 / 204)$ practicebased learning, 25.5\% (52/204) communication skills (Fig 1).

\section{Conclusion}

SIMBA is an effective virtual teaching model that improved clinicians' confidence in managing various conditions. Participants felt cases were relevant to their clinical practice and suited their personal learning style. Further work is warranted to explore whether increased confidence levels translated to better real-life performance. 


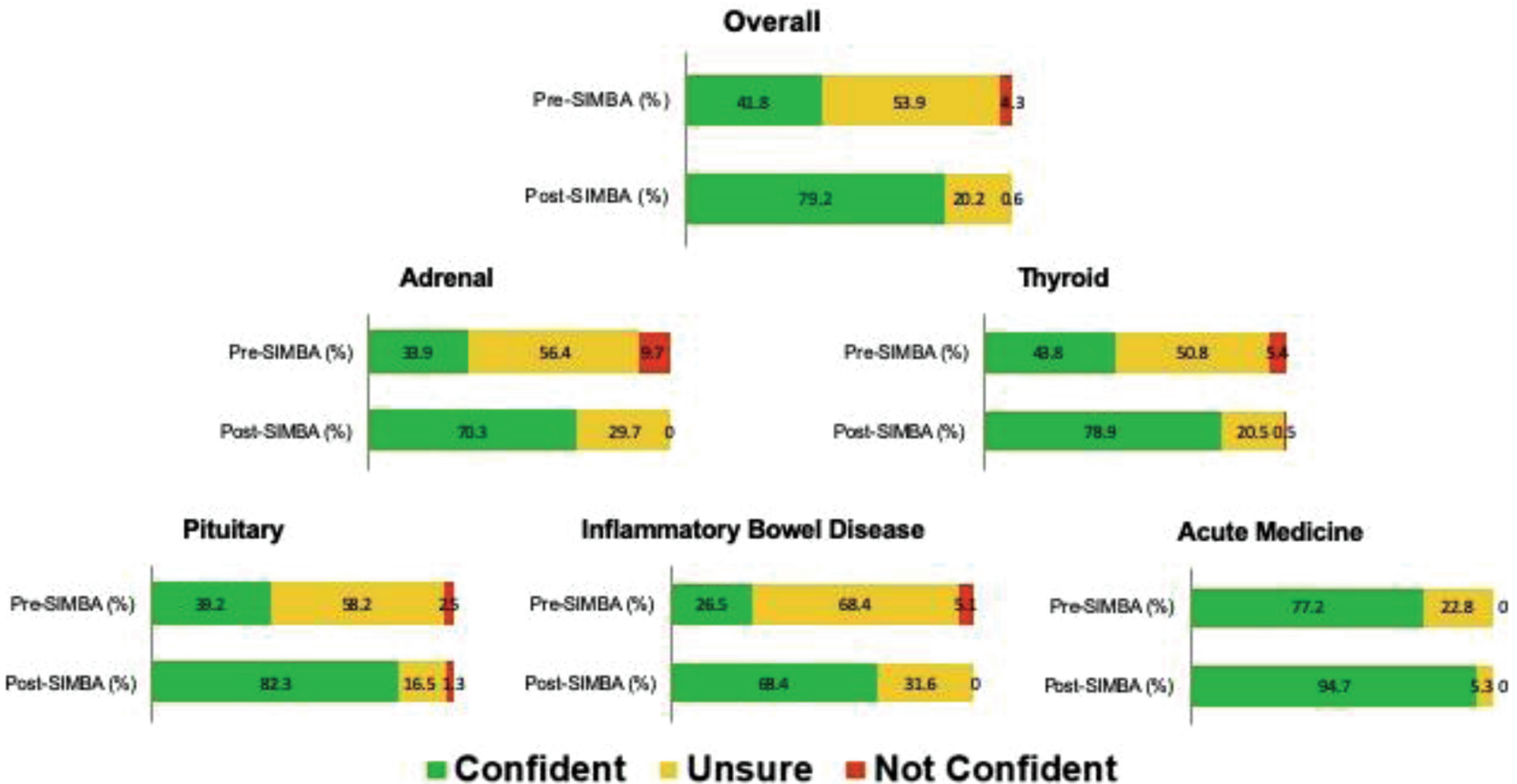

Fig 1. Change in self-reported confidence levels of participants' approach to various clinical scenarios pre- and post-SIMBA.

\section{Conflicts of interest}

None declared.

\section{References}

1 McGaghie WC, Issenberg SB, Petrusa ER et al. A critical review of simulation-based medical education research: 2003-2009. Med Educ 2010;44:50-63.
2 Melson E, Davitadze M, Aftab M et al. Simulation via instant messaging - Birmingham advance (SIMBA) model helped improve clinicians' confidence to manage cases in diabetes and endocrinology. BMC Med Educ 2020;20:274. 